\title{
ANALYTICAL AND NUMERICAL ANALYSES OF THE HEAT CONDUCTION OF CARBON NANOTUBES UNDER APPLIED VOLTAGES
}

\author{
Yahya ÖZ \\ Turkish Aerospace, R\&D Directorate, Advanced Composite Materials Technology Center \\ 06980 Kahramankazan, Ankara, yahya@unam.bilkent.edu.tr, ORCID: 0000-0003-3784-0495
}

(Geliş Tarihi: 14.02.2020, Kabul Tarihi: 14.01.2021)

\begin{abstract}
Electron field emission experiments of carbon nanotubes (CNT) show that due to heat CNTs breakdown faster under applied voltage than expected. Therefore, different systems with varying temperature distributions are considered analytically and numerically by use of the heat conduction equation. This methodology yields a phenomenological understanding and description of the thermoelectric behavior of CNTs under applied voltages.

Keywords: Carbon Nanotubes, Electron Field Emission, Heat Conduction.
\end{abstract}

\section{KARBON NANOTÜPLERIN ISI İLETIMININ UYGULANAN VOLTAJLAR ALTINDA ANALITIK VE SAYISAL ANALIZI}

Özet: Karbon nanotüplerin (KNT) elektron alanı emisyonu deneyleri, 1S1 nedeniyle KNT'lerin uygulanan voltaj altında beklenenden daha hızlı bozulduğunu göstermektedir. Bu nedenle, değişen sıcaklık dağılımlarına sahip farklı sistemler, 1S1 akışı denklemini kullanarak analitik ve sayısal olarak değerlendirilmektedir. Bu metodoloji, KNT'lerin termoelektrik davranışının uygulanan voltaj altında fenomenolojik davranışını ve tanımını sağlamaktadır.

Anahtar Kelimler: Karbon Nanotüp, Elektron Alan Emisyonu, Isı İletimi.

\section{NOMENCLATURE}

$U \quad$ Applied Voltage [V]

$k_{B} \quad$ Boltzmann Constant $[\mathrm{J} / \mathrm{K}]$

A Contact Area $\left[\mathrm{m}^{2}\right]$

I Current [A]

$j \quad$ Current Density $\left[\mathrm{A} / \mathrm{m}^{2}\right]$

$\sigma_{\text {el }} \quad$ Electrical Conductivity $[\mathrm{S} / \mathrm{m}]$

E Electric Field [V/m]

$\rho_{\mathrm{el}} \quad$ Electrical Resistivity $[\Omega \mathrm{m}]$

$q_{e} \quad$ Elementary Charge [C]

$\rho \quad$ Mass Density $\left[\mathrm{kg} / \mathrm{m}^{3}\right]$

$\vec{x} \quad$ Position Vector [m]

$R_{c} \quad$ Quality of Thermal Contact [K/W]

$r \quad$ Radius of a CNT $[\mathrm{m}]$

$c \quad$ Specific Heat $[\mathrm{J} /(\mathrm{kg} \mathrm{K})]$

$\sigma \quad$ Stefan-Boltzmann Constant $\left[\mathrm{W} /\left(\mathrm{m}^{2} \mathrm{~K}^{4}\right)\right]$

$T_{0} \quad$ Surrounding Temperature [K]

$T \quad$ Temperature [K]

$k \quad$ Thermal Conductivity [W/(m K)]

$t \quad$ Time [s]

$g \quad$ Volumetric Heat Source $\left[\mathrm{W} / \mathrm{m}^{3}\right]$

\section{INTRODUCTION}

Electron beams are involved in various applications and basic research tools like cathode ray tubes, x-ray tubes, scanning electron microscopes and transmission electron microscopes. Typically a high density of narrow electron beams is desired in such applications. Therefore, in contrast to electron guns that use thermionic emission of electrons from hot cathodes, which cause thermal broadening, cold cathodes are gaining interest. However, these cathodes need large electric fields, which yield migration of atoms at the tip surface. Hence, stable operation over long periods of time is quite difficult. However, CNTs offer a solution to this problem and are accordingly focused on (Bonard et.al, 2001; Cheng and Zhou, 2003; Ha et.al, 2013). Compared to metal or diamond tips, nanotube tips are inert and stable to long periods of operation. Moreover, other advantages like low threshold voltages for cold field emission, low temperature of operation, fast response times, low power and small sizes are available (de Jonge et.al, 2005; Charlier et.al, 2007). Many examples of these kinds of applications are known in the literature (de Heer et.al, 1995; Choi et.al, 1999; Sugie et.al, 2001; Croci et.al, 2004; Zhang et.al, 2005).

Due to the high electric fields, materials for field emission tips must be resistant to high temperatures caused by Joule heating and high stress. This framework may lead to the breakdown of the device. The breakdown occurs most likely at the point of the CNT where the temperature is at its maximum. 
The breakdown is usually completed in three consecutive phases. First, the device performance decreases. Afterwards, a structural failure arises in the form of a crack. Finally, the CNT is burned off (Wei et.al, 2007). Different models (Vincent et.al, 2002; Bocharov and Eletskii, 2013; Giubileo et.al, 2018) and experiments (Wang et.al, 2002; Bonard et.al, 2003; Fairchild et.al, 2019) were analyzed to understand the breakdown behavior of CNTs. The proposed theoretical models are based on Joule heating. The existence of two sources for the generation and dissipation of heat are assumed; electrical resistance (Joule heating) and heat radiation. The full solution of the heat conduction equation for this case is still an open question, because besides the advanced mathematical structure involved in the related equation, the temperature dependance of the resistivity and boundary conditions are needed (Collins et.al, 2001).

The goal of this article is the solution and investigation of the heat conduction equation with focus on applications of CNTs for electron field emission. The motivation for this research is two-fold. First, the systematic procedure for the analytic solution of the heat conduction equation with arbitrary boundary conditions and temperature dependance of the resistivity is introduced. The procedure is carried out using CNTs with constant boundary conditions as an example. Second, an efficient numerical algorithm (controlled by the analytical calculations) is used for the modeling of thermal dissipation in CNTs. In fact, possible solutions for the breakdown due to a self-heating process are analyzed.

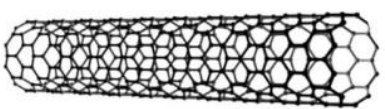

(a)

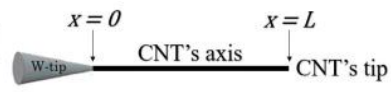

(b)
Figure 1. Schematic depiction of (a) single-walled CNT and (b) its associated field emission tip of length $L$ connected to a tungsten (W) tip. The configuration shows that the heat conduction is essentially one-dimensional.

\section{HEAT TRANSFER THROUGH CNT WITH BOUNDARY CONDITIONS}

The heat conduction equation in a non-uniform anisotropic medium is given by Eq. (1)

$$
\rho(\vec{x}) c(\vec{x}, t) \partial_{t} T(\vec{x}, t)=\nabla(k(\vec{x}, t) \nabla T(\vec{x}, t))+g(\vec{x}, t),
$$

where $\rho(\vec{x})$ is the mass density, $c(\vec{x}, t)$ the specific heat, $T(\vec{x}, t)$ the temperature distribution measured in $\mathrm{K}$, $k(\vec{x}, t)$ the thermal conductivity, $g(\vec{x}, t)$ the volumetric rate of internal energy generation, $t$ the time and $\vec{x}$ the position vector. Note that specific heat, thermal conductivity and volumetric heat source generally depend on the temperature and thus implicitly on position vector and time.
The configuration of the heat transfer problem considered is shown in Fig. 1.

As a result of the geometry of CNTs they are modeled as one-dimensional rods and Eq. (1) simplifies to its one-dimensional form. $\vec{x}$ and $\nabla$ are replaced with $x$ and $\partial_{x}$, respectively. However, the mathematical analysis in this article can in principle be used for arbitrary dimension $d$ if the need arises due to a use of another emitter in contrast to Fig. 1.

For the purpose of determining the volumetric heat source Ohm's law is used due to Joule heating which leads to

$$
j(x, t) E(x, t)=\frac{I^{2}}{A^{2} \sigma_{\mathrm{el}}(x, t)}=\frac{U^{2} \rho_{\mathrm{el}}(x, t)}{\left(\int_{0}^{L} \mathrm{~d} x \rho_{\mathrm{el}}(x, t)\right)^{2}},
$$

where $j(x, t)$ is the current density, $E(x, t)$ the electrical field, $\sigma_{\text {el }}(x, t)$ the electrical conductivity and $\rho_{\text {el }}(x, t)$ the electrical resistivity while the contact area is denoted with $A$. The Stefan-Boltzmann law which describes heat radiation as well as consideration of CNTs with lengths $L$ along the $x$-axis attached to a tungsten tip in combination with Eq. (2) yields Eq. (3)

$$
g(x, t)=\frac{U^{2} \rho_{\mathrm{el}}(x, t)}{\left(\int_{0}^{L} \mathrm{~d} x \rho_{\mathrm{el}}(x, t)\right)^{2}}-\frac{\sigma}{L}\left(T^{4}(x, t)-T_{0}^{4}\right)
$$

as expression for the volumetric heat source, where $U$ is the applied voltage and $\sigma$ the Stefan-Boltzmann constant. Furthermore, $T(0<x<L, 0)=T_{0}$ is assumed. For the purpose of defining the boundary conditions, the tips of the CNTs are considered. At the interface with the tungsten microtip a temperature drop due to the thermal contact resistance occurs. The microtip and surroundings temperatures are expressed as $T_{0}$ while $R_{c}$ represents the contact quality, i.e. the thermal contact resistance. These assumptions yield the boundary condition in Eq. (4)

$$
T(0, t)=T_{0}+\left.R_{c} A k(x, t) \partial_{x} T(x, t)\right|_{x=0} .
$$

Nevertheless, the interesting boundary is the free end of the nanotube. Electron emission on this end cools the tip since each electron carries energy $\frac{3}{2} k_{\mathrm{B}} T(L, t)$ due to classical statistical mechanics and the equipartition theorem (Tolman, 1918), where $k_{\mathrm{B}}$ is the Boltzmann constant. Hence, the heat flux can be written down by 
$\frac{3 k_{\mathrm{B}} I T(L, t)}{2 q_{e} A}$ with the elementary charge $q_{e}$. Note that in metals this effect is much smaller than radiative cooling. Contrarily, in CNTs it is a dominant factor due to high field emission current density and low surface area for radiative cooling (Chernozatonskii et.al, 1995; Rinzler et.al, 1995). Hence, the Stefan-Boltzmann law for radiation and Fourier's heat conduction relation yield Eq. (5)

$\left.\partial_{x} T(x, t)\right|_{x=L}=-\frac{\sigma}{k}\left(T^{4}(L, t)-T_{0}^{4}\right)-\frac{3 k_{B} I T(L, t)}{2 q_{e} A k(L, t)}$,

where $I$ is the current.

\section{Analytical Solution}

In the following mass density, specific heat and thermal conductivity are taken as constants for the analytic treatment of Eq. (1).

\section{Separation of variables}

Separation of variables is widely applied for the analytical solution of heat conduction problems. This section introduces the most general solution of the presented physical problem achievable by use of this method. Correspondingly, the Stefan-Boltzmann term in Eq. (3) and (5) is set to zero and Eq. (3) is assumed to be time-independent, since separation of variables is not applicable otherwise because the homogeneous boundary conditions for the Sturm-Liouville problem are not fulfilled. This corresponds to the assumptions that heat radiation is negligible and that Joule heating occurs instantaneously. In fact, Fig. 7 shows that the influence of time-dependent Joule heating is negligible in accordance with the literature (Wei et.al, 2007). Moreover, regarding the neglect of heat radiation it should be noted that this analytical approach is mainly used to control the numerical analysis. The numerical experiments are compared with the analytical results in Figs. 2-5. In Fig. 9, however, the heat radiation is also included numerically.

First, Eq. (1) is split into two simpler problems in Eq. (6) for the purpose of separation of variables; one steady state that is descibed by a nonhomogeneous ordinary differential equation with nonhomogeneous boundary conditions and one transient described by a homogeneous partial differential equation with homogeneous boundary conditions as well as a modified initial condition, i.e.

$T(x, t)=T_{\mathrm{H}}(x, t)+T_{\mathrm{SS}}(x)$.

Accordingly, one finds two separate problems formulated in Eqs. (7) and (8), i.e. $\partial_{x}^{2} T_{\mathrm{H}}(x, t) \quad=\frac{\rho c}{k} \partial_{t} T_{\mathrm{H}}(x, t)$,

$\left.R_{c} A k \partial_{x} T_{\mathrm{H}}(x, t)\right|_{x=0}=T_{\mathrm{H}}(0, t)$,

$\left.\partial_{x} T_{\mathrm{H}}(x, t)\right|_{x=L} \quad=-\frac{3 k_{\mathrm{B}} I}{2 q_{e} A k} T_{\mathrm{H}}(L, t)$

$T_{\mathrm{H}}(x, 0)$

$$
=T_{0}-T_{\mathrm{SS}}(x)
$$

and

$$
\begin{array}{ll}
\partial_{x}^{2} T_{\mathrm{SS}}(x) & =-\frac{g(x)}{k} \\
\left.R_{c} A k \partial_{x} T_{\mathrm{SS}}(x)\right|_{x=0} & =T_{\mathrm{SS}}(0)-T_{0} \\
\left.\partial_{x} T_{\mathrm{SS}}(x)\right|_{x=L} & =-\frac{3 k_{\mathrm{B}} I}{2 q_{e} A k} T_{\mathrm{SS}}(L)
\end{array}
$$

for $0 \leq x \leq L$ and $t \geq 0$.

Second, the steady state part in Eq. (8) can be solved trivially using traditional ordinary differential equation solution techniques, i.e. sum of the homogeneous and particular solutions. For this purpose $T_{\mathrm{SS}}(x)=T_{\mathrm{A}}(x)+T_{\mathrm{B}}(x)$ is used as an approach which leads to Eqs. (9) and (10)

$$
\begin{array}{ll}
\partial_{x}^{2} T_{\mathrm{A}}(x) & =0 \\
\left.R_{c} A k \partial_{x} T_{\mathrm{A}}(x)\right|_{x=0} & =T_{\mathrm{A}}(0)-T_{0}, \\
\left.\partial_{x} T_{\mathrm{A}}(x)\right|_{x=L} & =-\frac{3 k_{\mathrm{B}} I}{2 q_{e} A k} T_{\mathrm{A}}(L)
\end{array}
$$

and

$$
\begin{array}{ll}
\partial_{x}^{2} T_{\mathrm{B}}(x) & =-\frac{g(x)}{k}, \\
\left.R_{c} A k \partial_{x} T_{\mathrm{B}}(x)\right|_{x=0} & =T_{\mathrm{B}}(0), \\
\left.\partial_{x} T_{\mathrm{B}}(x)\right|_{x=L} & =-\frac{3 k_{\mathrm{B}} I}{2 q_{e} A k} T_{\mathrm{B}}(L) .
\end{array}
$$

Obviously, Eq. (9) yields Eq. (11)

$$
T_{\mathrm{A}}(x)=T_{0} \frac{L+\frac{2 q_{e} A k}{3 k_{\mathrm{B}} I}-x}{L+R_{c} A k+\frac{2 q_{e} A k}{3 k_{\mathrm{B}} I}}
$$

while Eq. (10) leads to an apparent particular solution in Eq. (12)

$$
T_{\mathrm{B}}(x)=-\int_{a}^{x} \mathrm{~d} x^{\prime} \int_{b}^{x^{\prime}} \frac{\mathrm{d} x^{\prime \prime}}{k} g\left(x^{\prime \prime}\right)
$$

where $a$ and $b$ follow from the homogeneous boundary conditions in Eq. (10) and are chosen such that Eqs. (13) hold 


$$
\begin{array}{ll}
R_{c} A(G(b)-G(0)) & =T_{\mathrm{B}}(0), \\
G(L)-G(b) & =\frac{3 k_{\mathrm{B}} I}{2 q_{e} A} T_{\mathrm{B}}(L),
\end{array}
$$

where $G(x)$ is the antiderivative of $g(x)$. In total, one finds Eq. (14)

$$
\begin{aligned}
T_{\mathrm{SS}}(x)= & \frac{T_{0}}{1+\frac{3 k_{\mathrm{B}} \lambda I A k}{2 q_{e} A k+3 k_{\mathrm{B}} L I}}-\frac{T_{0} x}{L+\lambda A k+\frac{2 q_{e} A k}{3 k_{\mathrm{B}} I}} \\
& -\int_{a}^{x} \mathrm{~d} x^{\prime} \int_{b}^{x^{\prime}} \frac{\mathrm{d} x^{\prime \prime}}{k} g\left(x^{\prime \prime}\right) .
\end{aligned}
$$

Note that this approach holds for any integrable volumetric heat source $g(x)$ and its antiderivative $G(x)$.

Third, the transient problem in Eq. (7) is handled by a separation into space-dependent and time-dependent functions of a single variable each, i.e. $T_{\mathrm{H}}(x, t)=\Psi(x) \Gamma(t)$. This produces two different ordinary differential equations given in Eqs. (15) and (16);

$$
\begin{array}{ll}
X^{\prime \prime}(x)+\lambda_{n}^{2} X(x) & =0, \\
R_{c} A k X^{\prime}(0)-X(0) & =0, \\
X^{\prime}(L)+\frac{3 k_{\mathrm{B}} I}{2 q_{e} A k} X(L) & =0
\end{array}
$$

and

$$
\Gamma^{\prime}(\mathrm{t})+\frac{k \lambda_{n}^{2}}{\rho c} \Gamma(t)=0 .
$$

Solving both equations separately, using the boundary conditions and recombining both solutions yields Eq. (17)

$$
T_{\mathrm{H}}(x, t)=\sum_{n=0}^{\infty} C_{n} \underbrace{\left(\lambda_{n} \cos \left(\lambda_{n} x\right)+\frac{\sin \left(\lambda_{n} x\right)}{R_{c} A k}\right)}_{=X_{n}(x)} e^{-\frac{k \lambda_{n}^{2} t}{c \rho}}
$$

with the roots $\lambda_{n}$ given by the transcendental Eq. (18)

$$
\tan \left(\lambda_{n} L\right)=\frac{\lambda_{n}+\frac{3 k_{\mathrm{B}} \lambda I \lambda_{n}}{2 q_{e}}}{R_{c} A k \lambda_{n}^{2}-\frac{3 k_{\mathrm{B}} I}{2 q_{e} A k}},
$$

and the constants $C_{n}$ determined in Eq. (19) by the nonhomogeneous initial condition of Eq. (7)

$$
C_{n}=\frac{2 R_{c} A k \int_{0}^{L} \mathrm{~d} x\left(T_{0}-T_{\mathrm{SS}}(x)\right) X_{n}(x)}{1+\left(1+\left(R_{c} A k \lambda_{n}\right)^{2}\right)\left(\frac{L}{R_{c} A k}+\frac{\frac{3 k_{B} I}{2 R_{c} q_{e}(A k)^{2}}}{\lambda_{n}^{2}+\left(\frac{3 k_{B} I}{2 q_{e} A k}\right)^{2}}\right)} .
$$

In total Eqs. (6), (14), (17)-(19) solve the formulated problem. The explicitness of the analytic solution should be noted. Obviously Eq. (19) can be simplified even further by inserting Eq. (14) and calculating the integral. Even though the expressions are lengthy, the calculations are straightforward and lead to Eq. (20)

$$
\begin{aligned}
C_{n}= & \frac{12 k_{B} R_{c} I T_{0} A^{2} k^{2}}{\lambda_{n}^{2}\left(2 q_{e} A k+3 k_{B} I\left(L+R_{c} A k\right)\right)} \\
& \left.\cdot\left(1+R_{c} A k \lambda_{n}^{2}\left(L+R_{c} A k\right)\right) \sin \left(L \lambda_{n}\right)-L \lambda_{n} \cos \left(L \lambda_{n}\right)\right) \\
& \cdot\left(1+\left(R_{c} A k \lambda_{n}\right)^{2}\right)\left(\begin{array}{c}
3 k_{B} I \\
q_{e}\left(\lambda_{n}^{2}+\left(\frac{3 k_{B} I}{2 q_{e} A k}\right)^{2}\right)
\end{array}\right) \\
& \left.+2 R_{c} A^{2} k^{2}\right)^{-1} .
\end{aligned}
$$

\section{Solution by Green's function}

Use of Green's function provides an efficient and straightforward method for constructing exact analytic solutions of various heat conduction problems. However, appropriate Green's functions must be available. The approach is used in mathematical physics for the solution of partial differential equations (Aizen et.al, 1974). Contrarily to the separation of variables, it is the most general method to solve nonhomogeneous, time-dependent conduction problems. Thus, a plane surface heat source of unit strength located at $x^{\prime}$ and an instantaneous heat source releasing its energy spontaneously at time $t^{\prime}$ into a zero temperature medium, both represented by Dirac delta functions, are considered for the auxiliary problem in Eq. (21) for $0<x<L$

$\frac{\rho c}{k} \partial_{t} G\left(x, t \mid x^{\prime}, t^{\prime}\right)=\partial_{x}^{2} G\left(x, t \mid x^{\prime}, t^{\prime}\right)+\frac{\delta\left(x-x^{\prime}\right) \delta\left(t-t^{\prime}\right)}{k}$

with boundary conditions in Eqs. (22)

$$
\begin{aligned}
& \left.R_{c} A k \partial_{x} G\left(x, t \mid x^{\prime}, t^{\prime}\right)\right|_{x=0}-G\left(0, t \mid x^{\prime}, t^{\prime}\right)=0, \\
& \left.\partial_{x} G\left(x, t \mid x^{\prime}, t^{\prime}\right)\right|_{x=L}+\frac{3 k_{B} I}{2 q_{e} A k} G\left(L, t \mid x^{\prime}, t^{\prime}\right)=0
\end{aligned}
$$

and initial condition $G\left(x, t \mid x^{\prime}, t^{\prime}\right)=0$ for $t<t^{\prime}$. The formulated auxiliary problem is quite useful since the 
solution of the original problem can be expressed just in terms of Green's function in Eq. (23)

$$
\begin{aligned}
T(x, t)= & T_{0} \int_{0}^{L} \mathrm{~d} x^{\prime} G\left(x, t \mid x^{\prime}, 0\right) \\
& +\frac{1}{\rho c} \int_{0}^{t} \mathrm{~d} t^{\prime} \int_{0}^{L} \mathrm{~d} x^{\prime} G\left(x, t \mid x^{\prime}, t^{\prime}\right) g\left(x^{\prime}, t^{\prime}\right) \\
& -\frac{T_{0}}{R_{c} A \rho c} \int_{0}^{t} \mathrm{~d} t^{\prime} G\left(x, t \mid 0, t^{\prime}\right) .
\end{aligned}
$$

Note the time-dependency in $g(x, t)$ in contrast to the method of separation of variables.

Typically Laplace transformations or the method of images are used for the determination of Green's function (Bilodeau, 1962). Instead, a general approach that uses seperation of variables is applied here. Therefore, the homogeneous version of the original problem with nonhomogeneous initial condition is considered. A separation in space- and time-dependent functions of a single variable each is assumed. The solution of this problem can be constructed in a simple manner in Eqs. (24) and (25) with the previously shown methodology. It is given by

$$
T(x, t)=\sum_{n=0}^{\infty} \tilde{C}_{n} X_{n}(x) e^{-\frac{k \lambda_{n}^{2} t}{c \rho}}
$$

and

$$
\tilde{C}_{n}=\frac{\frac{2 T_{0}}{\lambda_{n}}\left(1-\cos \left(\lambda_{n} L\right)+R_{c} A k \lambda_{n} \sin \left(\lambda_{n} L\right)\right)}{1+\left(1+\left(R_{c} A k \lambda_{n}\right)^{2}\right)} .
$$

Considering Eqs. (24) and (25) carefully, defining the norm in Eq. (26)

$$
\begin{aligned}
N_{n}^{-1} & =\int_{0}^{L} \mathrm{~d} x X_{n}^{2}(x) \\
& =\frac{2 R_{c} A k}{1+\left(1+\left(R_{c} A k \lambda_{n}\right)^{2}\left(\frac{L}{R_{c} A k}+\frac{\frac{3 k_{B} I}{2 R_{c} q_{e}(A k)^{2}}}{\lambda_{n}^{2}+\left(\frac{3 k_{B} I}{2 q_{e} A k}\right)^{2}}\right)\right.},
\end{aligned}
$$

that already appeared explicitly in Eq. (19) as well as (25), and using the property $G\left(x, t \mid x^{\prime}, t^{\prime}\right)=G\left(x, t-t^{\prime} \mid x^{\prime}, 0\right)$ as well as the Heaviside step function $H(x)$ yields Green's function

$G\left(x, t \mid x^{\prime}, t^{\prime}\right)=H\left(t-t^{\prime}\right) \sum_{n=0}^{\infty} \frac{X_{n}(x) X_{n}\left(x^{\prime}\right)}{N_{n}} e^{-\frac{k \lambda_{n}^{2}}{\rho c}\left(t-t^{\prime}\right)}$

Putting Eqs. (23) and (27) together produces the full analytic solution

$$
\begin{aligned}
T(x, t)=\sum_{n=1}^{\infty} \frac{X_{n}(x)}{N_{n}} e^{-\frac{k \lambda_{n}^{2}}{\rho c} t}\left(\left(T_{0}\left(\sin \left(\lambda_{n} L\right)+\frac{2-\cos \left(\lambda_{n} L\right)}{R_{c} A k \lambda_{n}}\right)\right.\right. \\
\left.+\int_{0}^{t} \mathrm{~d} t^{\prime} \int_{0}^{L} \frac{\mathrm{d} x^{\prime}}{\rho c} X_{n}\left(x^{\prime}\right) e^{\frac{k \lambda_{n}^{2}}{\rho c}} g\left(x^{\prime}, t^{\prime}\right)\right) \\
\left.-\frac{T_{0}}{R_{c} A k \lambda_{n}}\right)
\end{aligned}
$$

Note that the analytically derived Eq. (27) is applicable for all cases of volumetric heat source. Comparison with the originally formulated problem shows that just the Stefan-Boltzmann term in Eq. (5) is neglected. Hence, just the heat radiation at the free end is neglected in the analytical approach. Furthermore, the thermal conductivity is taken as constant. Investigations for a linear temperature dependence were performed in the literature (Zhang et.al, 2018).

\section{Numerical Analysis}

The numerical treatment of Eq. (1) is done by utilizing the temperature dependence of the electrical resistivity. Hence, for the volumetric heat source in Eq. (3) $g(x, t)=g(T(x, t))=g(T)$ is used, which corresponds to the expected physics, since the heat generation at location $x$ and time $t$ depends on the temperature at this location and time. The explicit dependance varies in different publications due to varying conduction mechanisms. Purcell (Purcell et.al, 2002) uses $\rho_{\mathrm{el}}(T)=\rho_{0}(1-\alpha T)$. This assumption takes hopping along thermally activated defect sites into account. This effect is well-known for large defect densities (Jang et.al, 2004; Dehghani et.al, 2012). The wave functions of electrons are highly localized in disordered systems which leads to a vanishing electrical cunductance at temperature zero. Nonetheless, electrons are hopping between localized states at finite temperatures resulting in electrical conduction. Hence, increasing temperature leads to a decreasing electrical resistivity. However, electron-phonon scatterings (scattering of electrons by the thermal motion of the lattice) should also be considered since the electrical resistivity $\rho_{\text {el }}(T)$ decreases indefinitely otherwise. Therefore, $\rho_{\mathrm{el}}(T)=\rho_{0}\left(1-\alpha_{1} T+\left(\alpha_{2} T\right)^{3 / 2}\right) \quad$ is used in this manuscript (Suzuura and Ando, 2002) while $\alpha_{1}$ and $\alpha_{2}$ 
are chosen such that they are fitting the experimental data.

The partial differential Eq. (1) is solved numerically by approximating it with difference equations while the derivatives are replaced with finite differences. Accordingly, this method leads to the recursive relation in Eq. (29)

$$
\begin{aligned}
T\left(x_{j}, t_{k+1}\right)= & T\left(x_{j}, t_{k}\right)+\frac{\Delta t}{\Delta x^{2} \rho\left(T\left(x_{j}, t_{k}\right)\right) c\left(T\left(x_{j}, t_{k}\right)\right)} \\
& \cdot\left(\partial_{T} \frac{k\left(T\left(x_{j}, t_{k}\right)\right)}{4}\left(T\left(x_{j+1}, t_{k}\right)-T\left(x_{j-1}, t_{k}\right)\right)^{2}\right. \\
& +k\left(T\left(x_{j}, t_{k}\right)\right)\left(T\left(x_{j-1}, t_{k}\right)-2 T\left(x_{j}, t_{k}\right)+T\left(x_{j+1}, t_{k}\right)\right) \\
& \left.+\Delta x^{2} g\left(T\left(x_{j}, t_{k}\right)\right)\right),
\end{aligned}
$$

where the temperature dependency of mass density, specific heat and thermal conductivity is used. Moreover, the evaluation is performed by utilization of the disretization $x_{1}, \ldots, x_{N}$ and $t_{1}, \ldots, t_{M}$ with $x_{j}=(j+1) \Delta x$ and $t_{k}=(k+1) \Delta t$, where $\Delta x$ and $\Delta t$ are step sizes. Due to the recursive relation the temperature at location $x_{j}$ and time $t_{k+1}$ depends on the temperatures at the locations $x_{j-1}, x_{j}, x_{j+1}$ and time $t_{k}$. The boundary conditions given in Eqs. (4) and (5) are valid for $x=x_{1}$ and $x=x_{N}$. C++ was used as programming language for the recursive solution of Eq. (29). This approach yields an efficient algorithm that is easy to implement (Fraser, 1909; Fornberg, 1988; Flajolet and Sedgewick, 1995; Zachos, 2008; Curtright and Zachos, 2013). However, instabilities for large time steps that disturb the data quality have to be treated carefully (Jaluria and Atluri, 1994). A von Neumann stability analysis for $g(T)=0$ yields $\frac{k \Delta t}{\rho c(\Delta x)^{2}} \leq \frac{1}{2}$ as a criterion for the stability (Charney et.al, 1950; Crank and Nicolson, 1996). In the numerical analysis $\frac{\Delta t}{(\Delta x)^{2}} \leq 1$ is used unless stated otherwise.

\section{RESULTS AND DISCUSSIONS}

For the sake of checking the obtained results and proving the usefulness of Eq. (28) numerical and analytic solutions are being compared. To test the numerical results with regard to their stability, rather obscure initial temperatures $T(0<x<L, 0)$ such as rectangular $\tilde{T} H\left(x-\frac{L}{4}\right) H\left(\frac{3 L}{4}-x\right)$, linear $\tilde{T} \frac{x}{L}$ or quadratic $\tilde{T}\left(\frac{x}{L}\right)^{2}$ expressions for $L=40 \cdot 10^{-6} \mathrm{~m}$ and $\tilde{T}=10 \mathrm{~K}$ are used. For simplicity the volumetric heat source $g(T)$ and the boundary condition $T(0, t)$ are set to zero in this numerical experiments. Furthermore, the method of images, i.e. $T\left(x_{N-1}, t\right)=T\left(x_{N+1}, t\right)$ is used for the boundary condition at $x=L$ in the numerical analysis. Hence, for these experiments the physically motivated Eqs. (3)-(5) are not considered.

All other constants are summarized in Table 1, in which physical parameters describing the behavior of multiwalled CNTs and field emission devices are taken from (Dai et.al, 1996; Ebbesen et.al, 1996; Hone et.al, 1999; Bachtold et.al, 1999; Yi et.al, 1999; Yang et.al, 2002; Milne et.al, 2003; Sveningsson et.al, 2004; Fujii et.al, 2005; Karim et.al, 2006; Karim et.al, 2007; Park et.al, 2010).

Table 1. Physical parameters of multiwalled nanotubes

\begin{tabular}{|l|c|}
\hline$T_{0}$ & $300 \mathrm{~K}$ \\
\hline$\lambda$ & $1.774 \cdot 10^{7} \mathrm{~K} / \mathrm{W}$ \\
\hline$U$ & $500-2000 \mathrm{~V}$ \\
\hline$r$ & $10^{-8} \mathrm{~m}$ \\
\hline$L$ & $0.5-40 \cdot 10^{-6} \mathrm{~m}$ \\
\hline$\rho$ & $1300 \mathrm{~kg} / \mathrm{m}^{3}$ \\
\hline$k$ & $100 \mathrm{~W} /(\mathrm{m} \mathrm{K})$ \\
\hline$c$ & $740 \mathrm{~J} /(\mathrm{kg} \mathrm{K})$ \\
\hline$\rho_{0}$ & $3.26 \cdot 10^{-5} \Omega \mathrm{m}$ \\
\hline$\alpha_{1}$ & $8.5 \cdot 10^{-4} \mathrm{~K}^{-1}$ \\
\hline$\alpha_{2}$ & $9.8 \cdot 10^{-6} \mathrm{~K}^{-1}$ \\
\hline
\end{tabular}

The results of the numerical experiments are shown in Figs. 2-5. Fig. 2 depicts the rectangular initial temperature $\tilde{T} H\left(x-\frac{L}{4}\right) H\left(\frac{3 L}{4}-x\right)$ as well as the analytical and numerical result after $10 n \mathrm{~s}$. The dots show the analytical solutions obtained by separation of variables in Eq. (6) as well as Green's function in Eq. (28). Moreover, Eq. (17) is considered two-fold. The sum is evaluated for the first ten and 100 summands, respectively. The dots therefore consist of a total of three different analytical solutions. Hence, every dot is actually a superposition of three different dots. It can be seen that both solution methods are equivalent as expected. In addition, the sums in Eqs. (17) and (28) converge fast due to the factor $e^{-\frac{k \lambda_{n}^{2}}{\rho c} t}$. For large times zero temperature is found everywhere along the CNT due to the factor $e^{-\frac{k \lambda_{n}^{2}}{\rho c} t}$ in Eq. (28). By virtue of the fixed boundary conditions, the curves obtained by the means of numerics agree with the analytical results at both ends. These curves were obtained for two different ratios, i.e. $\frac{\Delta t}{(\Delta x)^{2}}=0.1$ and $\frac{\Delta t}{(\Delta x)^{2}}=10$ and almost lie on top of each other. However, a discrepance between exact and numerical solutions can be observed in the middle of the CNT due to the discontinuous initial distribution. Accordingly, the numerical and analytical results agree in the other polynomial initial temperature cases for all times in Figs. 3-5. In analogy to Fig. 1, Fig. 
3 and Fig. 5 illustrate the linear initial conditions $\tilde{T} \frac{x}{L}$ and $\tilde{T}\left(\frac{x}{L}\right)^{2}$, respectively. The analytical result depicted with plus signs in Fig. 3 is obtained by separation of variables with 100 summands for $10 \mathrm{~ns}$. It is compared to two different numerical solutions due to varying ratios $\frac{\Delta t}{(\Delta x)^{2}}=0.1$ and $\frac{\Delta t}{(\Delta x)^{2}}=10$. The exact and numerical curves agree with each other. Fig. 5 emphasizes this result. The analytical solution obtained by Green's function with 100 summands completely fits the numerical result evaluated for $\frac{\Delta t}{(\Delta x)^{2}}=0.1$. Additionally, Fig. 4 shows the influence of time on the temperature distribution. The temperature distribution for the linear initial condition is obtained by Green's function and by numerics with $\frac{\Delta t}{(\Delta x)^{2}}=0.1$ for varying times, i.e., $5,10, \ldots, 50 n \mathrm{~s}$, and are in good agreement. As expected, the temperature along the CNT is decreasing for increasing times.

Fig. 6 shows the numerical result for the initial condition $T(0<x<L, 0)=T_{0}=300 \mathrm{~K}$ and $\frac{\Delta t}{(\Delta x)^{2}}=0.1$, but the inhomogeneities in the boundary conditions and in the heat conduction equation are neglected, i.e. the StefanBoltzmann term in Eq. (5) that describes heat radiation at the free end, the electron emission term of Eq. (5) and the volumetric heat source $g(T)$ are set to zero. As expected, the maximum temperature is reached at the free end since tip cooling effects are neglected. The time dependence of the temperature distribution is examined in the inset of Fig. 6. It can be seen that the steady-state is reached very fast, which was used as an assumption in previous publications (Huang et.al, 2004). However, the scale varies between $n$ s and $\mu$ s with varying lengths which can be seen in Fig. 7 .

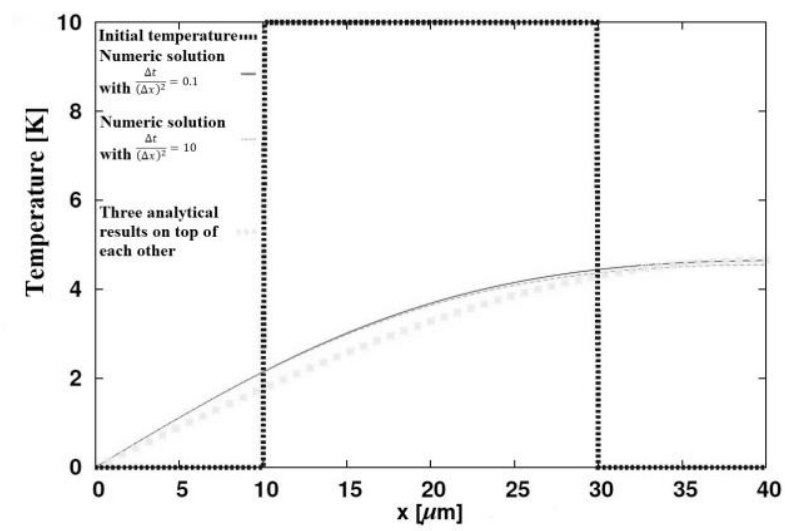

Figure 2. Temperature distribution at $10 n$ s along the $x$-axis for a rectangular initial condition denoted with the blue dashed line. Three different exact results obtained by separation of variables with ten and 100 summands and
Green's function with 100 summands are depicted as dots and lie on top of each. Furthermore, numerical results for $\frac{\Delta t}{(\Delta x)^{2}}=0.1$ and $\frac{\Delta t}{(\Delta x)^{2}}=10$ are illustrated with red and green lines and show good agreement with each other.

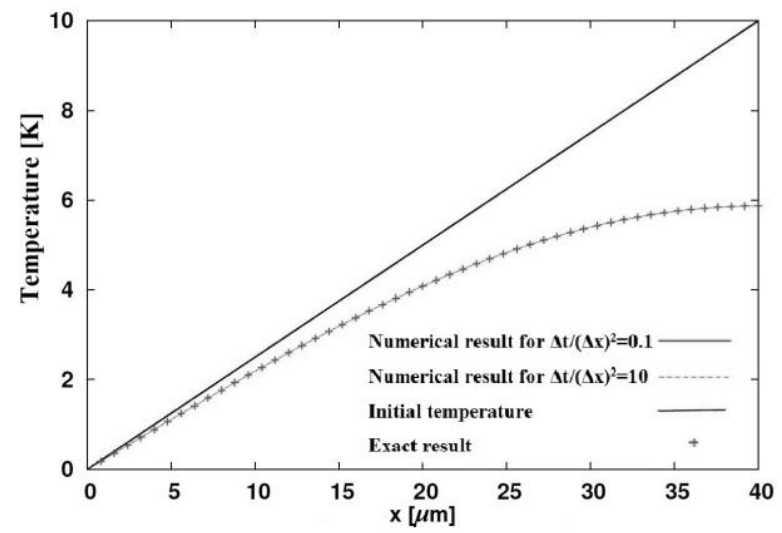

Figure 3. Temperature distribution at $10 n \mathrm{~s}$ along the $x$-axis for a linear initial condition illustrated with a blue line. As expected, numerical and analytic result are in good agreement even for large step sizes $\Delta t$. The error is of order $0.01 \%$.
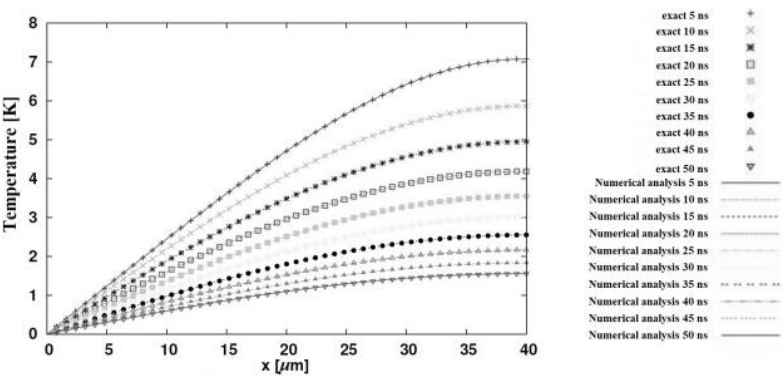

Figure 4. Temperature distribution along the $x$-axis for a linear initial condition and the corresponding time evolution. Exact and numerical solutions are observable. The temperature is decreasing for increasing time. Numerically, the steady-state temperature zero of this numerical experiment is found everywhere for $100 \mathrm{~ns}$. Hence, the time scale shows that the steady-state is reached rather fast.

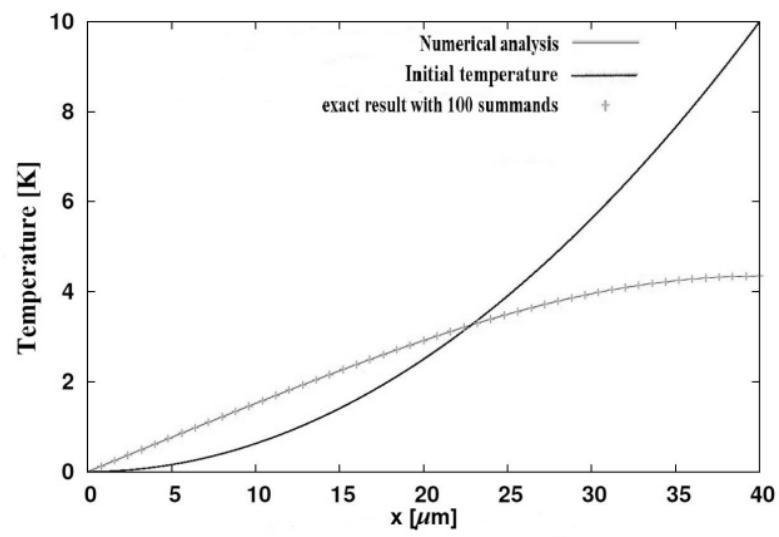

Figure 5. Temperature distribution at $10 n$ s along the $x$-axis for a quadratic initial condition illustated with a blue line. As expected, numerical and analytic result, i.e. red line and green plus signs, are in good agreement. 


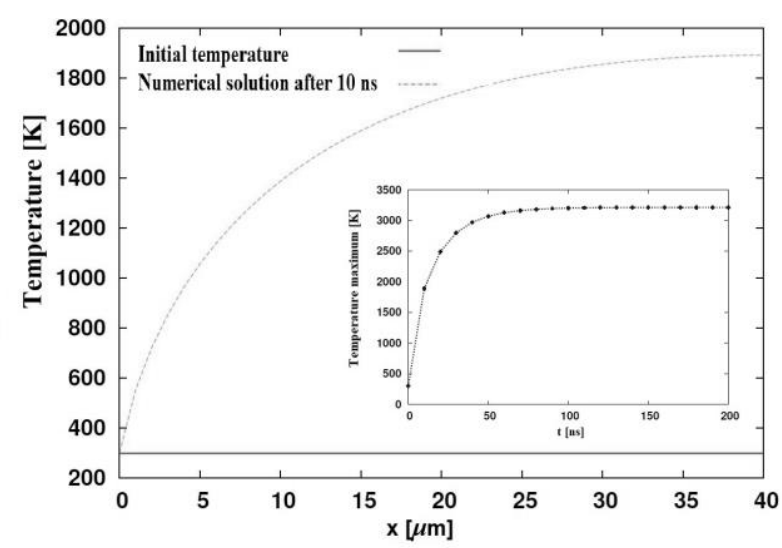

Figure 6. Temperature distribution after $10 \mathrm{n}$ s along the $x$ axis for initial condition $T_{0}=300 \mathrm{~K}$. The numerical solution was obtained for $\frac{\Delta t}{(\Delta x)^{2}}=0.1$. The inset shows the maximum temperature reached at the free end of the CNT in this configuration after several time intervals. The temperature at at the free end increases up to $3213 \mathrm{~K}$. This steady-state behavior is observable after approximately $100 \mathrm{~ns}$.

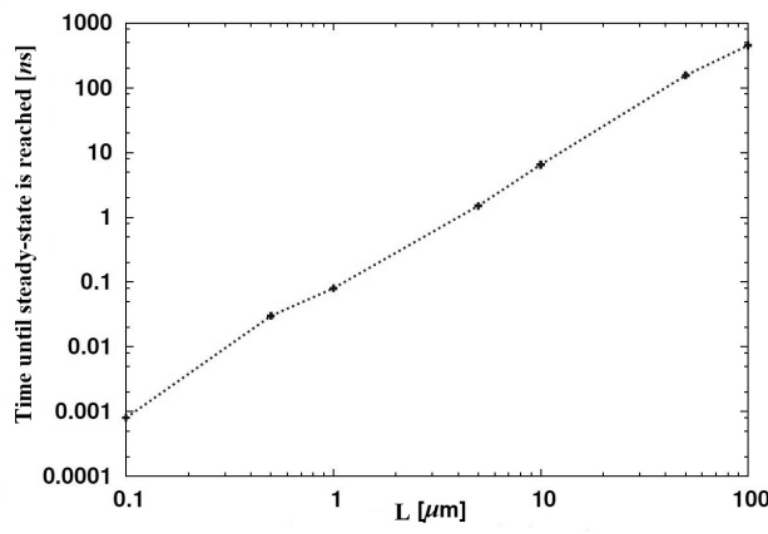

Figure 7. Time until the steady-state is reached for different CNT lengths. Growing lengths yield higher times.

Steady-state results of analytical evaluations with 100 summands and numerical calculations with $\frac{\Delta t}{(\Delta x)^{2}}=0.1$ are shown in Fig. 8 and 9, respectively. In addition to the temperature distribution, the influence of varying CNT lengths is also studied in these Figures.

The analytical solution takes all terms in Eqs. (3)-(5) with the exception of the Stefan-Boltzmann term in Eq. (5) into account and uses the CNT length $L=5 \cdot 10^{-7} \mathrm{~m}$ as well as $U=2000 \mathrm{~V}$ as voltage. Note that the StefanBoltzmann term occuring in the volumetric heat source in Eq. (3) is considered. However, for the evaluation of Eq. (28) in combination with Eq. (3) and temperaturedependent electrical resistivity $\rho_{\mathrm{el}}(T)$ discretization as well as iteration is used. For fixed parameters given in Table 1 the maximum temperature is not reached at the free end of the CNT due to the electrons carrying energy. Hence, a tip cooling effect is observed in Fig. 8.

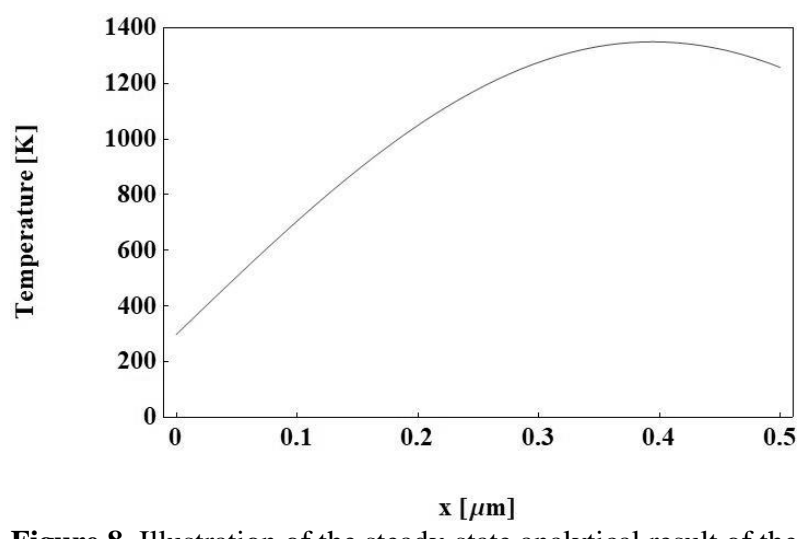

Figure 8. Illustration of the steady-state analytical result of the original problem for a CNT of $5 \cdot 10^{-7} \mathrm{~m}$ while just the Stefan-Boltzmann term in Eq. (5) is neglected.

In contrast to the analytical treatment, the numerical analysis takes all terms in Eqs. (3)-(5) into account. Moreover, the CNT length $L=4 \cdot 10^{-5} \mathrm{~m}$ and $500 \mathrm{~V}$ for the voltage is used.

A similar but increased tip cooling effect is observed in the numerical calculations since in this case the in the analytic calculations neglected term of the boundary condition in Eq. (5) is taken into account. This additional heat radiation at the free end of the CNT leads to a greater decrease in temperature at this end if Fig. 8 and 9 are compared.

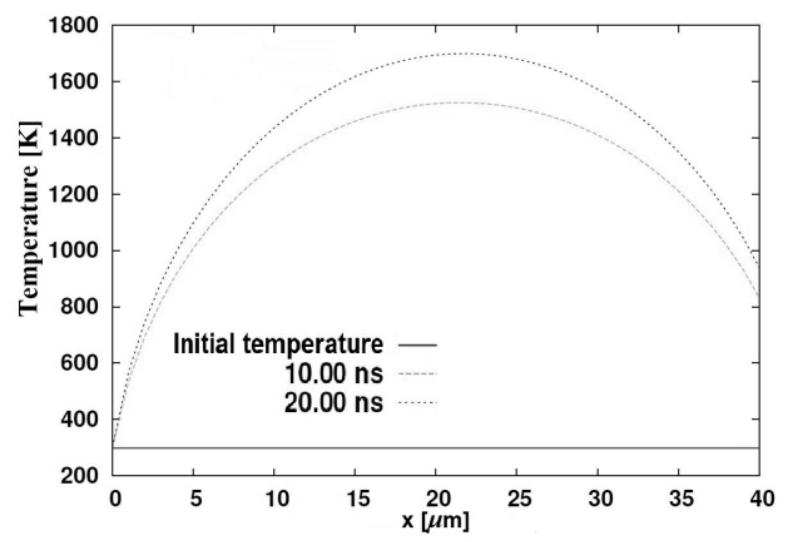

Figure 9. Numerically calculated temperature distribution for the original problem along the CNT with length $4 \cdot 10^{-5} \mathrm{~m}$, where $\frac{\Delta t}{(\Delta x)^{2}}=0.1$ was used.

Notwithstanding this subtle difference, both analytical and numerical calculations predict breakdown of the CNT during electron field emission some space away of the free tip since it occurs most likely at the point of the CNT where the temperature is at its maximum. In fact, this is in good agreement with experiments (Liu et.al, 2019; Lin et.al, 2019).

Note that comparison of both results shows different maximum temperatures for different CNT lengths. Shortening the length decreases the maximum temperature, which is an important mechanism for the 
stability of the CNT field emission tip. This observation is strengthened by different numerical experiments. However, in comparison with Fig. 9 a higher voltage according to Table 1 was applied in Fig. 8 for the purpose of demonstrating the breakdown behavior with just analytical results and proving the existence of the experimentally observed threshold voltage at which breakdown occurs (Bonard et.al, 2002; Doytcheva et.al, 2006).

Another strategy for preventing the breakdown behavior are alternating voltages that are set to zero in certain time intervals. For this purpose Eq. (30)

$U(t)=U_{0} \sum_{n \in \aleph_{0}} H\left(t-n t_{1}\right) H\left(n t_{1}+t_{2}-t\right)$

is used as voltage, where $U_{0}=2000 \mathrm{~V}, t_{1}=100 \mathrm{~ns}$ and $t_{2}=20 \mathrm{~ns}$. Note that for this analysis time steps $\Delta t$ have to be smaller by at least an order than the period $\Delta t$ of the alternating voltage due to the NyquistShannon sampling theorem (Shannon, 1949). Furthermore, the period $t_{1}$ should be large enough since the physical system needs time to adjust. Hence, $\Delta t=\frac{t_{1}}{10}$ is used besides $\frac{\Delta t}{(\Delta x)^{2}}=0.1$ and Table 1 for this purpose. Moreover, for simplicity, heat radiation is neglected in Eq. (3) and (5) for this numerical evaluation. These assumptions lead to a periodic cooling and heating of the CNT in Fig. 10. This method might be a possible solution for the avoidance of breakdown. In fact, this strategy is in the focus of experiments (Zhang et.al, 2020).

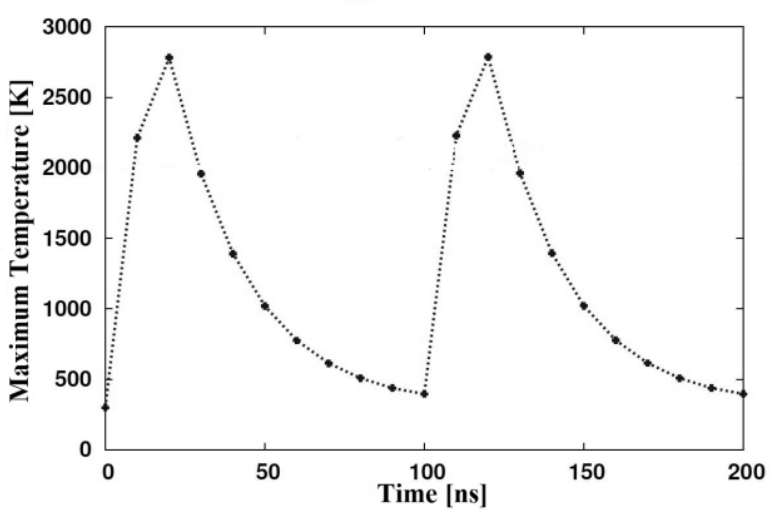

Figure 10. Maximum temperature of CNTs under applied alternating voltages

\section{CONCLUSIONS}

In summary, the thermal behavior of CNTs during electron field emission was analyzed by construction of analytical solutions of the heat conduction equation as well as numerical experiments. The influence of different inhomogeneities encoded in the volumetric heat source $g(x, t)$ in Eq. (1) as well as initial and boundary conditions in Eqs. (4)-(5) was analyzed. In contrast to prior publications the time-dependence and the Stefan-Boltzmann term in the boundary condition of the free end as well as the contact quality were taken into account. Nevertheless, temperature dependent thermal conductivity, quantum size effects (Sandomirskiı̌, 1967) and stresses in axial as well as radial direction that occur due to the electrostatic force were neglected. However, this simplification still yields good agreement with experiments. The breakdown mechanism of CNTs under applied voltages is explained completely by use of Joule heating, heat radiation and electron emission at the tip. In particular, this study was able to confirm the experimental knowledge that the breakdown does not occur at the end of the CNT but rather a short distance beforehand due to tip cooling caused by electron emission.

\section{ACKNOWLEDGEMENTS}

The author acknowledges financial support by the Scientific and Technological Research Council of Turkey within the support program 1515 and 1004 for research and laboratory developments within the projects 5189901 and 20AG001, respectively. Moreover, the author is grateful to the Institute of Materials Science and Nanotechnology at Bilkent University and Department of Engineering Sciences at Middle East Technical University for providing the needed infrastructure.

\section{REFERENCES}

Aizen A. M., Redchits I. S. and Fedotkin I. M., 1974, On improving the convergence of series used in solving the heat-conduction equation, Journal of Engineering Physics, 26, 453-458.

Bachtold A., Strunk C., Salvetat J., Forró L., Nussbaumer T. and Schönenberger, 1999, AharonovBohm oscillations in carbon nanotubes, Nature, 397, 673-675.

Bilodeau G. G., 1962, The Weierstrass transform and Hermite polynomials, Duke Mathematical Journal, 29, 293-308.

Bocharov G. S. and Eletskii A. V., 2013, Theory of Carbon Nanotube (CNT)-Based Electron Field Emitters, Nanomaterials, 3, 393-442.

Bonard J., Dean K. A., Coll B. F. and Klinke C., 2002, Field Emission of Individual Carbon Nanotubes in the Scanning Electron Microscope, Physical Review Letters, 89, 197602.

Bonard J., Kind H., Stöckli T. and Nisson L., 2001, Field emission from carbon nanotubes: the first five years, Solid-State Electronics, 45, 893-914. 
Bonard J., Klinke C., Dean K. A. and Coll B. F., 2003, Degradation and failure of carbon nanotube field emitters, Physical Review B, 67, 115406.

Charlier J., Blasé X. and Roche S., 2007, Electronic and transport properties of nanotubes, Reviews of Modern Physics, 79, 677-732.

Charney J. G., Fjörtoft R. and J. Von Neumann, 1950, Numerical Integration of the Barotropic Vorticity Equation, Tellus, 2, 237-254.

Cheng Y. and Zhou O., 2003, Electron field emission from carbon nanotubes, Comptes Rendus Physique, 4, 1021-1033.

Chernozatonskii L. A., Gulyaev Y. V., Kosakovskaja Z. J., Sinitsyn N. I., Torgashov G. V., Zakharchenko Y. F., Fedorov E. A. and Val'chuk V. P., 1995, Electron field emission from nanofilament carbon films, Chemical Physics Letters, 233, 63-68.

Choi W. B., Chung D. S., Kang J. H., Kim H. Y., Jin Y. W., Han I. T., Lee Y. H., Jung J. E., Lee N. S., Park G. S. and Kim J. M., 1999, Fully sealed, high-brightness carbon-nanotube field-emission display, Applied Physics Letters, 75, 3129-3131.

Collins P. G., Hersam M., Arnold M., Martel R. and Avouris P., 2001, Current Saturation and Electrical Breakdown in Multiwalled Carbon Nanotubes, Physical Review Letters, 86, 3128-3131.

Crank J. and Nicolson P., 1996, A practical method for numerical evaluation of solutions of partial differential equations of the heat-conduction type, Advances in Computational Mathematics, 6, 207-226.

Croci M., Arfaoui I., Stöckli T., Chatelain A. and Bonard J., 2004, A fully sealed luminescent tube based on carbon nanotube field emission, Microelectronics Journal, 35, 329-336.

Curtright T. L. and Zachos C. K., 2013, Umbral Vade Mecum, Frontiers in Physics, 1, 15.

Dai H., Wong E. W. and Lieber C. M., 1996, Probing Electrical Transport in Nanomaterials: Conductivity of Individual Carbon Nanotubes, Science, 272, 523-526.

de Heer W. A., Châtelain A. and Ugarte D., 1995, A Carbon Nanotube Field-Emission Electron Source, Science, 270, 1179-1180.

de Jonge N., Allioux M., Oostveen J. T., Teo K. B. K. and Milne W. I., 2005, Optical Performance of CarbonNanotube Electron Sources, Physical Review Letters, 94, 186807.

Dehghani S., Moravvej-Farshi M. K. and Sheikhi M. H., 2012, Temperature dependence of electrical resistance of individual carbon nanotubes and carbon nanotubes network, Modern Physics Letters B, 26, 1250136.

Doytcheva M., Kaiser M. and de Jonge N., 2006, In situ transmission electron microscopy investigation of the structural changes in carbon nanotubes during electron emission at high currents, Nanotechnology, 17, 32263233 .

Ebbesen T. W., Lezec H. J., Hiura H., Bennett J. W., Ghaemi H. F. and Thio T., 1996, Electrical conductivity of individual carbon nanotubes, Nature, 382, 54-56.

Fairchild S. B., Zhang P., Park J., Back T. C., Marincel D., Huang Z. and Pasquali M., 2019, Carbon Nanotube Fiber Field Emission Array Cathodes, IEEE Transactions on Plasma Science, 47, 2032-2038.

Flajolet P. and Sedgewick R., 1995, Mellin transforms and asymptotics: Finite differences and Rice's integrals, Theoretical Computer Science, 144, 101-124.

Fornberg B., 1988, Generation of finite difference formulas on arbitrarily spaced grids, Mathematics of Computation, 51, 699-706.

Fraser, D. C., 1909, On the Graphic Delineation of Interpolation Formulæ, Journal of the Institute of Actuaries, 43, 235-241.

Fujii M., Zhang X., Xie H., Ago H., Takahashi K., Ikuta T., Abe H. and Shimizu T., 2005, Measuring the Thermal Conductivity of a Single Carbon Nanotube, Physical Review Letters, 95, 065502.

Giubileo F., Di Bartolomeo A., Iemmo L., Luongo G. and Urban F., 2018, Field Emission from Carbon Nanostructures, Applied Sciences, 8, 526.

Ha J. M., Kim H. J., Raza H. S. and Cho S. O., 2013, Highly stable carbon nanotube field emitters on small metal tips against electrical arcing, Nanoscale Research Letters, 8, 355.

Hone J., Whitney M., Piskoti C. and Zettl A., 1999, Thermal conductivity of single-walled carbon nanotubes, Physical Review B, 59, R2514-R2516.

Huang N. Y., She J. C., Deng S. Z., Xu N. S., Bishop H., Huq S. E., Wang L., Zhong D. Y., Wang E. G. and Chen D. M., 2004, Mechanism Responsible for Initiating Carbon Nanotube Vacuum Breakdown, Physical Review Letters, 93,075501.

Jaluria Y. and Atluri S. N., 1994, Computational heat transfer, Computational Mechanics, 14, 385-386.

Jang W. Y., Kulkarni N. N., Shih C. K. and Yao Z., 2004, Electrical characterization of individual carbon 
nanotubes grown in nanoporous anodic alumina templates, Applied Physics Letters, 84, 1177-1179.

Karim S., Toimil-Molares M. E., Balogh A. G., Ensinger W., Cornelius T. W., Khan E. U. and Neumann R., 2006, Morphological evolution of $\mathrm{Au}$ nanowires controlled by Rayleigh instability, Nanotechnology, 17, 5954-5959.

Karim S., Toimil-Molares M. E., Ensinger W., Balogh A. G., Cornelius T. W., Khan E. U. and Neumann R., 2007, Influence of crystallinity on the Rayleigh instability of gold nanowires, Journal of Physics D: Applied Physics, 40, 3767-3770.

Lin C., Xiang Y. and Zhang J., 2019, Post-breakdown field emission behavior of a planar-structured single SWNT bundle in air, Journal of Micromechanics and Microengineering, 29, 065005.

Liu Z., Zhang Y., Zhao P., Ye Y., Chen J., Xu N. and Deng S., 2019, In situ study of field emission vacuum breakdown of individual multi-wall carbon nanotube, Micro \& Nano Letters, 14, 206-210.

Milne W. I., Teo K. B. K., Chhowalla M., Amaratunga G. A. J., Lee S. B., Hasko D. G., Ahmed H., Groening O., Legagneux P., Gangloff L., Schnell J. P., Pirio G., Pribat D., Castignolles M., Loiseau, Semet V., Binh V. T., 2003, Electrical and field emission investigation of individual carbon nanotubes from plasma enhanced chemical vapour deposition, Diamond and Related Materials, 12, 422-428.

Park S., Chae S., Rhee J. and Kang S., 2010, A Study on Electrical and Thermal Properties of Polyimide/MWNT Nanocomposites, Bulletin of the Korean Chemical Society, 31, 2279-2282.

Purcell S. T., Vincent P., Journet C. and Binh V. T., 2002, Hot Nanotubes: Stable Heating of Individual Multiwall Carbon Nanotubes to $2000 \mathrm{~K}$ Induced by the Field-Emission Current, Physical Review Letters, 88, 105502.

Rinzler A. G., Hafner J. H., Nikolaev P., Nordlander P., Colbert D. T., Smalley, R. E., Lou L., Kim S. G. and Tománek D., 1995, Unraveling Nanotubes: Field Emission from an Atomic Wire, Science, 269, 15501553.

Sandomirskiı̌ V. B., 1967, Quantum Size Effect in a Semimetal Film, Soviet Physics Journal of Experimental and Theoretical Physics, 25, 101-106.

Shannon C. E., 1949, Communication in the Presence of Noise, Proceedings of the IRE, 37, 10-21.

Sugie H., Tanemura M., Filip V., Iwata K., Takahashi K. and Okuyama, F., 2001, Carbon nanotubes as electron source in an x-ray tube, Applied Physics Letters, 78, 2578-2580.

Suzuura H. and Ando T., 2002, Phonons and electronphonon scattering in carbon nanotubes, Physical Review $B, 65,235412$.

Sveningsson M., Morjan R. E., Nerushev O. and Campbell E. E. B., 2004, Electron field emission from multi-walled carbon nanotubes, Carbon, 42, 1165-1168.

Tolman R. C., 1918, A General Theory of Energy Partition with Applications to Quantum Theory, Physical Review, 11, 261-275.

Vincent P., Purcell S. T., Journet C. and Binh V. T., 2002, Modelization of resistive heating of carbon nanotubes during field emission, Physical Review B, 66, 075406.

Wang Z. L., Gao R. P., de Heer W. A. and Poncharal P., 2002, In situ imaging of field emission from individual carbon nanotubes and their structural damage, Applied Physics Letters, 80, 856-858.

Wei W., Liu Y., Wei Y., Jiang K., Peng. L. and Fan S., 2007, Tip Cooling Effect and Failure Mechanism of Field-Emitting Carbon Nanotubes, Nano Letters, 7, 6468.

Yang D. J., Zhang Q., Chen G., Yoon S. F., Ahn J., Wang S. G., Zhou Q., Wang Q. and Li J. Q., 2002, Thermal conductivity of multiwalled carbon nanotubes, Physical Review B, 66, 165440.

Yi W., Lu L., Dian-lin Z., Pan Z. W. and Xie S. S., 1999, Linear specific heat of carbon nanotubes, Physical Review B, 59, R9015-R9018.

Zachos C. K., 2008, Umbral deformations on discrete space-time, International Journal of Modern Physics A, 23, 2005-2014.

Zhang P., Park J., Fairchild S. B., Lockwood N. P., Lau Y. Y., Ferguson J. and Back T., 2018, Temperature Comparison of Looped and Vertical Carbon Nanotube Fibers during Field Emission, Applied Sciences, 8, 1175 .

Zhang Y., Tan Y., Wang L., Li B., Ke Y., Liao M., Xu N., Chen J. and Deng S., 2020, Electron emission and structure stability of carbon nanotube cold cathode driven by millisecond pulsed voltage, Vасииm, 172, 109071.

Zhang J., Yang G., Cheng Y., Gao B., Qiu Q., Lee Y. Z., Lu J. P. and Zhou O., 2005, Stationary scanning Xray source based on carbon nanotube field emitters, Applied Physics Letters, 86, 184104. 


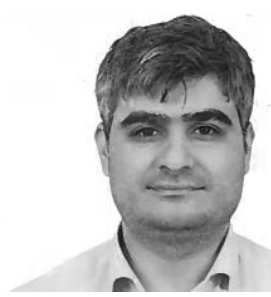

Yahya Öz is the Chief Engineer of the Advanced Composite Materials Technology Center of the R\&D Directorate at Turkish Aerospace, a researcher in the Institute of Materials Science and Nanotechnology at Bilkent University and a Visiting Assistant Professor in the Department of Engineering Sciences at the Middle East Technical University, Ankara, Turkey. He received his B.Sc. (2011) in Applied Physics and Chemistry, his M.Sc. (2013) and his Ph.D. (2017) in Physics in the Faculty of Mathematics and Natural Sciences at the University of Wuppertal, Germany, where he constructed and solved the Hubbard model with impurity at arbitrary temperature as well as manipulated the host's density of states by keeping integrability. Following his $\mathrm{PhD}$, he became a postdoctoral researcher and worked on transport properties of integrable systems as well as the hydrodynamic approach for integrable systems out of equilibrium. In 2019, he joined Turkish Aerospace. His current research interests include integrable models, modeling of nanomaterials as well as nanocomposites properties and modeling, fabrication and experimentation of composite materials for aerospace applications. Dr. Yahya Öz is the recipient of the full scholarship (2010-2017) by Germany's largest, oldest and most prestigious scholarship foundation, the German Academic Scholarship Foundation, and the 2014 Outstanding Young Scientist Award by the German Association for the Promotion of Mathematics and Natural sciences. 